\title{
Maternal and child outcomes reported by breastfeeding women following mRNA COVID- 19 vaccination
}

3 Kerri Bertrand ${ }^{1}$, Gordon Honerkamp-Smith ${ }^{1}$ and Christina Chambers ${ }^{1,2}$

4 Affiliations: Department of Pediatrics ${ }^{1}$, and Herbert Wertheim School of Public Health and

5 Longevity Science ${ }^{2}$, University of California, San Diego, La Jolla, CA 92093

6 Short Title: Breastfeeding outcomes after COVID-19 vaccination

7 Address Correspondences to:

Kerri Bertrand

Department of Pediatrics

University of California, San Diego

9500 Gilman Drive, \#0828, La Jolla, CA 92093

Telephone 8582461713

Email: kdutcher@health.ucsd.edu

\section{Conflict of Interest Disclosures}

Dr. Chambers receives research funding from the following industry sponsors and a foundation: Amgen Inc.; AstraZeneca; Celgene; GlaxoSmithKline; Janssen Pharmaceuticals; Pfizer, Inc.; Regeneron; Hoffman La-Roche-Genentech; Genzyme Sanofi-Aventis; Takeda Pharmaceutical Company Limited; Sanofi; UCB Pharma, USA; Sun Pharma Global FZE; Gilead; and the Gerber Foundation.

\section{Funding/Support}

Funding for this study was in part provided by a supplement to NIH-NICHD R21 Grant Number 1R21HD104412-01 to Christina Chambers 


\section{Table of Contents Summary}

\section{What's known on this subject:}

One published U.S. study with 31 breastfeeding women who were vaccinated with either of the two available mRNA COVID-19 vaccines found that $67 \%$ experienced any side effects after dose one and $61 \%$ after dose two of the vaccine.

\section{What this study adds:}

34 More than $85 \%$ of 180 breastfeeding women who received an mRNA COVID-19 vaccine 35 reported local or systemic symptoms, with higher frequency following the second dose. Few symptoms were reported in their breastfed children. No serious adverse events were noted.

\section{Contributors' Statement Page}

Dr. Chambers and Ms. Bertrand designed the study and supervised the collection of data used in the study. Mr. Honerkamp-Smith performed the statistical analysis. All authors were involved in preparing the manuscript. All authors approved the final manuscript as submitted and agree to be accountable for all aspects of the work.

\section{Acknowledgements}

The authors wish to thank student researchers Angelique Ghadishah, Bianca Kermani, Samantha Powell, and Zoe Sidiropoulos for helping with data collection. We also wish to express our appreciation to the women who participated in this study for giving so generously of their time and effort to provide clinical information. 
medRxiv preprint doi: https://doi.org/10.1101/2021.04.21.21255841; this version posted April 25, 2021. The copyright holder for this preprint (which was not certified by peer review) is the author/funder, who has granted medRxiv a license to display the preprint in perpetuity.

It is made available under a CC-BY-NC-ND 4.0 International license .

\section{INTRODUCTION}

Clinical trials for the both the Pfizer-BioNTech BNT162b2 and Moderna mRNA-1273

COVID-19 vaccines demonstrated ability to prevent infection and severe disease, leading to emergency use authorization by the U.S. Food and Drug Administration., ${ }^{1,2}$

The American College of Obstetrics and Gynecology and The Society for Maternal Fetal

Medicine have recommended that these mRNA vaccines be made available for lactating women.

However, initial trials excluded breastfeeding women, leading to questions about their safety. ${ }^{3}$

57

One study of 31 breastfeeding women who received an mRNA vaccine found that $>60 \%$

reported side effects. ${ }^{4}$ We sought to evaluate a larger sample of vaccinated breastfeeding women and their breastfed children.

\section{MATERIALS AND METHODS}

Breastfeeding women in the U.S. who received an mRNA vaccine were enrolled into the Mommy’s Milk Human Milk Research Biorepository at the University of California, San Diego. Demographics, health history, vaccine brand, and maternal symptoms and child events were collected by maternal interview and questionnaire for seven days following each dose. The study was approved by the UC San Diego IRB; written consent was obtained from participants. Maternal and child characteristics and outcomes were compared by brand for each dose using Student's t-test for continuous and Fisher's exact test for categorical variables. Missing values were excluded. $\mathrm{R}$ version 25 4.0.3 was used for analyses.

\section{Results}

Between December 14, 2020 and February 1, 2021, 180 women who received either mRNA vaccine were enrolled. As shown in Table 1, 128 women $(71.1 \%)$ received the Pfizer vaccine and $52(28.9 \%)$ received the Moderna brand. Child age at enrollment averaged 7.47 
medRxiv preprint doi: https://doi.org/10.1101/2021.04.21.21255841; this version posted April 25, 2021. The copyright holder for this preprint (which was not certified by peer review) is the author/funder, who has granted medRxiv a license to display the preprint in perpetuity.

It is made available under a CC-BY-NC-ND 4.0 International license .

73 months (SD 5.44, Range 0.09 to 27.45 months). The majority (86.0\%) were exclusively

74 breastfed.

Following dose one, similar proportions of women reported any vaccine symptom by

76 brand (89.4\% Pfizer; 98.1\% Moderna); frequency by specific symptom did not differ by brand.

77 However, following dose two, women who received the Moderna brand were significantly more

78 likely to report systemic side effects including chills, muscle/body aches, fever, vomiting. They

79 were also more likely to report localized symptoms including pain, redness, swelling or itching

80 at the injection site than women following dose two of the Pfizer brand (all p's <0.05). A small

81 proportion of women following dose one of either vaccine reported a reduction in milk supply.

82 There was a significant difference in reduction of milk supply following dose two by brand

83 (8.0\% vs. $23.4 \%$ for Pfizer and Moderna, respectively, $\mathrm{p}<0.05)$. However, in all cases milk

84 production returned to normal within 72 hours. Three women reported a change in color of milk

85 (blue-green) following dose one; two reported change in color of milk following dose two (Table $862)$.

Few events were reported for children following maternal vaccination with either brand or either dose. The most common child events following dose two were irritability (10.3\% and

$8910.4 \%$ for Pfizer and Moderna, respectively), poor sleep (7.8\% and 8.3\% for Pfizer and

90 Moderna respectively), with significantly more drowsiness reported for children whose mothers

91 received Moderna vs. Pfizer (0\% vs. 6.4\%, p=0.02) (Table 2). 
medRxiv preprint doi: https://doi.org/10.1101/2021.04.21.21255841; this version posted April 25, 2021. The copyright holder for this preprint

(which was not certified by peer review) is the author/funder, who has granted medRxiv a license to display the preprint in perpetuity.

It is made available under a CC-BY-NC-ND 4.0 International license .

\section{Discussion}

We found $>85 \%$ of participants reported any symptoms for both the Pfizer-BioNTech and

94 Moderna vaccines following either dose. This is substantially higher than the 61-67\% of women

95 who reported any symptom in the Gray et al. paper. ${ }^{4}$ This could be due to differences in methods

96 of assessment, timing, and number of symptoms specifically queried. However, consistent with

97 adult participants in clinical trials for each vaccine, we noted increased frequencies of most

98 symptoms following the second dose compared to the first. ${ }^{5,6}$ We also found significantly greater

99 frequencies of localized pain, redness, swelling and itching at the injection site as well as

100 systemic symptoms including chills, muscle/body aches, fever and vomiting following dose two

101 of the Moderna brand vs. Pfizer. Some participants reported a reduction in milk supply, which in

102 all cases returned to normal. We found low frequencies of any events reported in children, all of

103 which were non-serious. To our knowledge there have been no previous studies published on

104 outcomes in breastfed children.

These data are reassuring regarding the safety of vaccination in breastfeeding women and 106 their breastfed children with either of the mRNA COVID-19 vaccines. Additional studies are

107 underway to evaluate milk composition and antibody status in samples obtained from women

108 participating in the current study. 
medRxiv preprint doi: https://doi.org/10.1101/2021.04.21.21255841; this version posted April 25, 2021. The copyright holder for this preprint (which was not certified by peer review) is the author/funder, who has granted medRxiv a license to display the preprint in perpetuity. It is made available under a CC-BY-NC-ND 4.0 International license.

\section{References}

1. FDA authorizes Pfizer-BioNTech COVID-19 vaccine. Med Lett Drugs Ther. Jan 11 2021;63(1615):1-2.

2. $\quad$ FDA authorizes Moderna COVID-19 vaccine. Med Lett Drugs Ther. Jan 25 2021;63(1616):9-10.

3. Adhikari EH, Spong CY. COVID-19 Vaccination in Pregnant and Lactating Women. JAMA. Mar 16 2021;325(11):1039-1040. doi:10.1001/jama.2021.1658

4. Gray KJ, Bordt EA, Atyeo C, et al. COVID-19 vaccine response in pregnant and lactating women: a cohort study. Am J Obstet Gynecol. Mar 24 2021;doi:10.1016/j.ajog.2021.03.023

5. National Center for Immunization and Respiratory Diseases. Local Reactions, Systemic Reactions, Adverse Events, and Serious Adverse Events: Pfizer-BioNTech COVID-19 Vaccine. Updated 12/13/2020. Accessed 04/16, 2021. https://www.cdc.gov/vaccines/covid-19/info-byproduct/pfizer/reactogenicity.html

6. National Center for Immunization and Respiratory Diseases. Local Reactions, Systemic Reactions, Adverse Events, and Serious Adverse Events: Moderna COVID-19 Vaccine. Accessed 04/16, 2021. https://www.cdc.gov/vaccines/covid-19/info-by-product/moderna/reactogenicity.html 
medRxiv preprint doi: https://doi.org/10.1101/2021.04.21.21255841; this version posted April 25, 2021. The copyright holder for this preprint (which was not certified by peer review) is the author/funder, who has granted medRxiv a license to display the preprint in perpetuity.

It is made available under a CC-BY-NC-ND 4.0 International license .

Table 1: Characteristics of breastfeeding women who received either mRNA COVID-19 vaccine and their breastfed children, $\mathrm{N}=180$

\begin{tabular}{|c|c|c|c|}
\hline & $\begin{array}{l}\text { Pfizer/BioNTech } \\
\mathrm{n}=128\end{array}$ & $\begin{array}{l}\text { Moderna } \\
\mathrm{n}=\mathbf{5 2}\end{array}$ & $\begin{array}{l}\text { All } \\
\mathrm{N}=180\end{array}$ \\
\hline Maternal Age (years) & $34.92(3.67)$ & $34.50(3.24)$ & $34.80(3.54)$ \\
\hline \multicolumn{4}{|l|}{ Race } \\
\hline White & $119(93.7)$ & $39(75.0)$ & $159(88.3)^{* *}$ \\
\hline Black & $0(0.0)$ & $1(1.9)$ & $1(0.6)$ \\
\hline Asian & $7(5.5)$ & $12(23.1)$ & $19(10.6)$ \\
\hline Native American/ Alaska Native & $1(0.8)$ & $0(0.0)$ & $1(0.6)$ \\
\hline \multicolumn{4}{|l|}{ Ethnicity } \\
\hline Non-Hispanic & $123(96.9)$ & $51(98.1)$ & $174(96.7)$ \\
\hline Hispanic & $4(3.1)$ & $1(1.9)$ & $6(3.3)$ \\
\hline \multicolumn{4}{|l|}{ Education } \\
\hline Some College & $1(0.8)$ & $0(0.0)$ & $1(0.6)$ \\
\hline College Graduate & $33(26.2)$ & $12(23.5)$ & $45(25.3)$ \\
\hline Post-Graduate & $92(73.0)$ & $39(76.5)$ & $132(74.2)$ \\
\hline \multicolumn{4}{|l|}{ Income per year } \\
\hline$\$ 10,001-\$ 49,999$ & $0(0.0)$ & $1(2.0)$ & $1(0.6)$ \\
\hline$>\$ 60,000$ & $127(100.0)$ & $50(98.0)$ & $178(99.4)$ \\
\hline Full Time Employment & $89(71.8)$ & $34(66.7)$ & $124(70.5)$ \\
\hline \multicolumn{4}{|l|}{ Maternal Co-Morbidities } \\
\hline $\mathrm{BMI}>30$ & $16(12.6)$ & $4(7.8)$ & $20(11.2)$ \\
\hline Hypertension & $2(1.6)$ & $0(0.0)$ & $2(1.1)$ \\
\hline Diabetes & $0(0.0)$ & $0(0.0)$ & $0(0.0)$ \\
\hline Asthma & $20(15.7)$ & $6(11.5)$ & $26(14.4)$ \\
\hline Prior COVID-19 infection & $4(3.1)$ & $4(7.7)$ & $8(4.4)$ \\
\hline Child Age in months (SD, Range) & $7.60(5.54,0.39-27.45)$ & $7.16(5.26,0.10-23.45)$ & $7.47(5.44,0.09-27.45)$ \\
\hline \multicolumn{4}{|l|}{ Child Sex } \\
\hline Female & $75(59.5)$ & $25(49.0)$ & $100(56.7)$ \\
\hline Male & $51(40.5)$ & $26(51.0)$ & $77(43.3)$ \\
\hline Pre-term Delivery & $5(3.9)$ & $4(7.7)$ & $9(5.0)$ \\
\hline Exclusive Breastfeeding & $107(84.3)$ & $45(90.0)$ & $153(86.0)$ \\
\hline
\end{tabular}

${ }^{*} \mathrm{p}<0.05 * * \mathrm{p}<0.005$. Continuous variables were summarized by mean (SD) and group comparisons were made using a t-test. Categorical variables were summarized by count $(\%)$ and group comparisons were made using Fisher's Exact test. 
Table 2. Symptoms reported by breastfeeding women who received either mRNA COVID-19 vaccine and events in their breastfed children

\begin{tabular}{|c|c|c|c|c|c|c|c|c|}
\hline \multirow[b]{3}{*}{ Symptom } & \multicolumn{4}{|c|}{ Within 7 days of Dose 1} & \multicolumn{4}{|c|}{ Within 7 days of Dose 2} \\
\hline & \multicolumn{2}{|c|}{ Pfizer-BioNTech } & \multicolumn{2}{|c|}{ Moderna } & \multicolumn{2}{|c|}{ Pfizer-BioNTech } & \multicolumn{2}{|c|}{ Moderna } \\
\hline & $\begin{array}{l}\text { Women } \\
n=126\end{array}$ & $\begin{array}{l}\text { Children } \\
\mathrm{n}=129\end{array}$ & $\begin{array}{l}\text { Women } \\
\mathrm{n}=52\end{array}$ & $\begin{array}{l}\text { Children } \\
\mathrm{n}=53\end{array}$ & $\begin{array}{l}\text { Women } \\
\mathrm{n}=123\end{array}$ & $\begin{array}{l}\text { Children } \\
n=126\end{array}$ & $\begin{array}{l}\text { Women } \\
\mathbf{n}=52\end{array}$ & $\begin{array}{l}\text { Children } \\
\text { n=53 }\end{array}$ \\
\hline Any Maternal Symptoms & $110(89.4)$ & & $51(98.1)$ & & $114(98.3)$ & & $50(100.0)$ & \\
\hline Any Local Symptoms & $105(86.8)$ & & $50(96.2)$ & & $102(87.9)$ & & $48(98.0)^{*}$ & \\
\hline Pain at Injection Site & $105(86.8)$ & & $50(96.2)$ & & $102(87.9)$ & & $48(98.0)$ & \\
\hline Redness at Injection Site & $7(5.9)$ & & $5(9.6)$ & & $3(2.6)$ & & $15(31.9)^{* *}$ & \\
\hline Swelling at Injection Site & $7(5.9)$ & & $6(11.5)$ & & $7(6.1)$ & & $14(29.8)^{* *}$ & \\
\hline Itching at Injection Site & $3(3.5)$ & & $4(7.7)$ & & $5(4.4)$ & & $8(17.4)^{*}$ & \\
\hline Any Systemic Symptoms & $51(41.5)$ & & $28(53.8)$ & & $101(87.1)$ & & $48(96.0)^{*}$ & \\
\hline Chills & $8(6.8)$ & & $5(9.6)$ & & $55(47.8)$ & & $36(75.0)^{* *}$ & \\
\hline Headache & $28(23.7)$ & & $13(25.0)$ & & $74(64.3)$ & & $35(71.4)$ & \\
\hline Joint Pain & $7(5.9)$ & & $1(1.9)$ & & $31(27.2)$ & & $16(34.0)$ & \\
\hline Muscle/Body Aches & $16(13.6)$ & & $6(11.5)$ & & $71(61.7)$ & & $41(83.7)^{*}$ & \\
\hline Fatigue or Tiredness & $31(26.3)$ & & $12(23.1)$ & & $82(71.3)$ & & $42(84.0)$ & \\
\hline Fever & $1(0.8)$ & & $2(3.8)$ & & $28(24.3)$ & & $23(46.9)^{*}$ & \\
\hline Nausea & $5(4.2)$ & & $1(1.9)$ & & $18(15.7)$ & & $13(27.7)$ & \\
\hline Vomiting & $0(0.0)$ & & $0(0.0)$ & & $1(0.9)$ & & $4(8.5)^{*}$ & \\
\hline Diarrhea & $2(1.7)$ & & $0(0.0)$ & & $3(2.6)$ & & $3(6.4)$ & \\
\hline Abdominal Pain & $1(0.8)$ & & $0(0.0)$ & & $4(3.4)$ & & $3(6.4)$ & \\
\hline Rash (body) & $1(0.8)$ & & $1(1.9)$ & & $1(0.9)$ & & $1(2.1)$ & \\
\hline Other & $7(5.9)$ & & $3(5.8)$ & & $7(6.3)$ & & $3(6.8)$ & \\
\hline \multicolumn{9}{|l|}{ Change in Milk Supply } \\
\hline More Milk & $4(3.3)$ & & $0(0.0)$ & & $4(3.6)$ & & $3(6.4)$ & \\
\hline Less Milk & $9(7.3)$ & & $6(11.5)$ & & $9(8.0)$ & & $11(23.4)^{*}$ & \\
\hline Change in Milk Color & $2(8.0)$ & & $1(7.1)$ & & $1(4.0)$ & & $1(6.2)$ & \\
\hline \multicolumn{9}{|l|}{ Child Events } \\
\hline Drowsiness & & $0(0.0)$ & & $1(2.0)$ & & $0(0.0)$ & & $3(6.4)^{*}$ \\
\hline Sedation & & $0(0.0)$ & & $0(0.0)$ & & $0(0.0)$ & & $0(0.0)$ \\
\hline Poor feeding & & $0(0.0)$ & & $0(0.0)$ & & $0(0.0)$ & & $1(2.1)$ \\
\hline Rash & & $1(0.8)$ & & $0(0.0)$ & & $1(0.9)$ & & $1(2.1)$ \\
\hline Bruising/bleeding & & $0(0.0)$ & & $0(0.0)$ & & $0(0.0)$ & & $0(0.0)$ \\
\hline Constipation & & $0(0.0)$ & & $1(2.0)$ & & $1(0.9)$ & & $1(2.1)$ \\
\hline Diarrhea & & $1(0.8)$ & & $2(3.9)$ & & $3(2.6)$ & & $1(2.1)$ \\
\hline Stools w/ abnormal color & & $1(0.8)$ & & $2(3.9)$ & & $2(1.7)$ & & $2(4.2)$ \\
\hline Fever & & $2(1.7)$ & & $0(0.0)$ & & $2(1.7)$ & & $0(0.0)$ \\
\hline Low Body Temp & & $0(0.0)$ & & $0(0.0)$ & & $0(0.0)$ & & $0(0.0)$ \\
\hline Restlessness & & $1(0.8)$ & & $0(0.0)$ & & $4(3.4)$ & & $2(4.2)$ \\
\hline Irritability & & $2(1.7)$ & & $2(3.9)$ & & $12(10.3)$ & & $5(10.4)$ \\
\hline Poor sleep & & $4(3.4)$ & & $3(5.9)$ & & $9(7.8)$ & & $4(8.3)$ \\
\hline High-pitched crying & & $0(0.0)$ & & $0(0.0)$ & & $0(0.0)$ & & $0(0.0)$ \\
\hline Abnormal movements & & $0(0.0)$ & & $0(0.0)$ & & $0(0.0)$ & & $0(0.0)$ \\
\hline Abnormal skin color & & $1(0.8)$ & & $0(0.0)$ & & $0(0.0)$ & & $0(0.0)$ \\
\hline
\end{tabular}

